\title{
Dirac Equation on the Torus and Rationally Extended Trigonometric Potentials within Supersymmetric QM
}

\author{
Özlem Yeşiltaş \\ Department of Physics, Faculty of Science, Gazi University, 06500 Ankara, Turkey \\ Correspondence should be addressed to Özlem Yeşiltaş; yesiltas@gazi.edu.tr
}

Received 21 September 2017; Accepted 17 January 2018; Published 18 February 2018

Academic Editor: Burak Bilki

Copyright (C) 2018 Özlem Yeșiltaş. This is an open access article distributed under the Creative Commons Attribution License, which permits unrestricted use, distribution, and reproduction in any medium, provided the original work is properly cited. The publication of this article was funded by SCOAP

\begin{abstract}
The exact solutions of the $(2+1)$-dimensional Dirac equation on the torus and the new extension and generalization of the trigonometric Pöschl-Teller potential families in terms of the torus parameters are obtained. Supersymmetric quantum mechanics techniques are used to get the extended potentials when the inner and outer radii of the torus are both equal and inequal. In addition, using the aspects of the Lie algebraic approaches, the iso $(2,1)$ algebra is also applied to the system where we have arrived at the spectrum solutions of the extended potentials using the Casimir operator that matches with the results of the exact solutions.
\end{abstract}

\section{Introduction}

There are several potentials which are used in both theoretical and applied physics with exact solutions in nonrelativistic quantum mechanics. These potential classes can also give physical results in the successful union of quantum mechanics and special relativity where the Dirac equation has a successful explanation on the antimatter, spin, and the realistic behavior of atoms [1]. On the other hand, the gravitational field effects on some quantum mechanical systems have been studied as an exciting research field [24]. From the symmetries of the Dirac equation [5] and Hermiticity and uniqueness [6] to the factorization and pseudosupersymmetry $[7,8]$, covariant form of the Dirac equation and its aspects are studied. The gravitational background can bring some mathematical difficulties through some geometries. One of them is the solutions of the wave equations on the torus geometry [9-12]. At the same time, in physical applications, such as graphene related ones, it is stated that the curvature of the material can change the electron density of the states. In [9], graphene nanoribbon along the surface of a torus is examined within the long wave approximation where the Dirac equation is solved approximately. Then, the recent study aims to bring a new viewpoint to the problem of the exact solutions for the Dirac equation on the torus which may lead to both applied and theoretical interest in the recent studies. When considering the works which are about the general theory of relativity and the quantum mechanics unification, there is a fact that the curvature of spacetime at the position of the atom can affect the spectrum. Therefore, the problem of electron and its perturbed energy spectrum owing to the gravitational field can bring more problems in the solutions through the geometries of the spacetime. Moreover, there is a class of potentials known as rationally extended potential models that have attracted much attention $[13,14]$. The extension of the theory within the exceptional polynomials for the Dirac systems is first studied using the Darboux transformations [15]. Hence, this work also involves the rationally extended potentials which can be suitable for the Hamiltonian obtained after torus parametrization.

In this paper, Section 2 involves the relativistic quantum mechanical wave equation in the gravitational background for a massless fermion where the Fermi velocity is taken as a position dependent function [16]. Supersymmetric partner potentials are obtained for the transformed Dirac Hamiltonian. The spectrum and spinor solutions are given when the inner and outer radii of the torus are equal and inequal. In Section 3, we present the iso $(2,1)$ Lie algebraic computations for the Dirac Hamiltonians. 


\section{Dirac Equation on the Torus}

Formulating the Dirac equation in curved spacetime, it is known that all metrics related by a general coordinate transformation are physically equivalent and physical observables in gravitation field should be invariant under general coordinate transformations. This is known as general covariance principle which necessitates transforming a tensor in the flat spacetime as a tensor under general transformations in the curved manifold. The covariant generalization of the Dirac equation to curved space was independently introduced by Weyl and by Fock $[17,18]$. Then, the Dirac equation can be written in terms of vierbein fields and gravitational spin connection as [17]

$$
\left[i \gamma^{\mu}\left(\partial_{\mu}-\Gamma_{\mu}\right)\right] \Psi=0
$$

where $\Gamma_{\mu}$ is the spin connection and $\Psi$ is the spinor which includes electron's wave-functions $\Psi=\left(\begin{array}{l}\psi_{1} \\ \psi_{2}\end{array}\right)$ near the Dirac point. The Dirac matrices $\gamma^{\mu}$ in curved spacetime satisfy

$$
\left\{\gamma^{\mu}, \gamma^{\nu}\right\}=2 g^{\mu \nu}
$$

Here $g^{\mu \nu}$ is the metric tensor and the tetrad(vierbein) frames field is defined as

$$
g_{\mu \nu}=e_{\mu}^{a} e_{\nu}^{b} \eta_{a b}
$$

where $\eta_{a b}=\operatorname{diag}\left[\begin{array}{lll}1 & -1 & -1\end{array}\right]$. The metric for the torus surface is given by

$$
d s^{2}=d t^{2}-a^{2} d v^{2}-(c+a \cos v)^{2} d u^{2}
$$

In the metric given above, the inner radius of the torus is $c$, the outer radius is given by $a$, and $u, v \in[0,2 \pi)$. We can use the spin connection formula which is

$$
\Gamma_{\mu}=\frac{1}{4} \gamma_{a} \gamma_{b} e_{\lambda}^{a} g^{\lambda \sigma}\left(\partial_{\mu} e_{\sigma}^{b}-\Gamma_{\mu \sigma}^{\lambda} e_{\lambda}^{b}\right)
$$

where $\Gamma_{\mu \sigma}^{\lambda}$ are the Christoffel symbols. In [9], the symbols $\Gamma_{\mu \sigma}^{\lambda}$ were given in terms of the variable $R\left(x_{1}\right)$ which corresponds to $R(v)$ in our work. So one can obtain them as

$$
\begin{aligned}
& \Gamma_{12}^{2}=-\frac{a \sin v}{c+a \cos v}, \\
& \Gamma_{22}^{1}=\frac{1}{a}(c+a \cos v) \sin v
\end{aligned}
$$

and accordingly,

$$
\begin{aligned}
& \Gamma_{0}=0, \\
& \Gamma_{1}=0, \\
& \Gamma_{2}=-\gamma_{1} \gamma_{2} \frac{a \sin v}{2(c+a \cos v)} .
\end{aligned}
$$

For the Dirac matrices, we will use $\sigma_{j}$ Pauli matrices as $\gamma_{0}=$ $\sigma_{3}, \gamma_{1}=-i \sigma_{2}$, and $\gamma_{2}=-i \sigma_{1}$. We use (6) and (7) in (1) and we get [9]

$$
\begin{aligned}
& \left(V_{F}^{-1} \partial_{t}\right) \psi_{1}=\left(-\frac{1}{a^{2}} \partial_{v}-\frac{i}{R^{2}} \partial_{u}+\frac{R^{\prime}}{2 R^{2} a}\right) \psi_{2} \\
& \left(V_{F}^{-1} \partial_{t}\right) \psi_{2}=\left(-\frac{1}{a^{2}} \partial_{v}+\frac{i}{R^{2}} \partial_{u}+\frac{R^{\prime}}{2 R^{2} a}\right) \psi_{1},
\end{aligned}
$$

where $R=c+a \cos v$. We note that $\partial_{0}=V_{F}^{-1} \partial_{t}$ is taken and the Fermi velocity $V_{F}^{-1}$ may not be a constant [16]. Using $\left(\begin{array}{l}\psi_{1}(t, v, u) \\ \psi_{2}(t, v, u)\end{array}\right)=e^{i E t-i k u}\left(\begin{array}{l}\psi_{1}(v) \\ \psi_{2}(v)\end{array}\right)$, we obtain

$$
\begin{aligned}
& -\frac{d^{2} \psi_{1}}{d x^{2}}+\frac{a V_{F}(x) R^{\prime}(x)-R(x)^{2} V_{F}^{\prime}(x)}{V_{F}(x) R(x)^{2}} \frac{d \psi_{1}}{d x} \\
& +\left(\frac{k^{2} a^{4}}{R^{4}(x)}-\frac{2 k R^{\prime}(x) a^{2}}{R(x)^{3}}-\frac{R^{\prime}(x)^{2} a^{2}}{4 R(x)^{4}}-\frac{R^{\prime}(x)^{2} a}{R(x)^{3}}\right. \\
& \left.+\frac{k V_{F}^{\prime}(x) a^{2}}{R(x)^{2} V_{F}(x)}+\frac{R^{\prime}(x) V_{F}^{\prime}(x) a}{2 R(x)^{2} V_{F}(x)}+\frac{R^{\prime \prime}(x) a}{2 R(x)^{2}}\right) \\
& \cdot \psi_{1}(x)=\frac{E^{2} a^{4}}{V_{F}(x)^{2}} \psi_{1}(x) \\
& -\frac{d^{2} \psi_{2}}{d x^{2}}+\frac{a V_{F}(x) R^{\prime}(x)-R(x)^{2} V_{F}^{\prime}(x)}{V_{F}(x) R(x)^{2}} \frac{d \psi_{2}}{d x} \\
& +\left(\frac{k^{2} a^{4}}{R^{4}(x)}+\frac{2 k R^{\prime}(x) a^{2}}{R(x)^{3}}-\frac{R^{\prime}(x)^{2} a^{2}}{4 R(x)^{4}}-\frac{R^{\prime}(x)^{2} a}{R(x)^{3}}\right. \\
& \left.-\frac{k V_{F}^{\prime}(x) a^{2}}{R(x)^{2} V_{F}(x)}+\frac{R^{\prime}(x) V_{F}^{\prime}(x) a}{2 R(x)^{2} V_{F}(x)}+\frac{R^{\prime \prime}(x) a}{2 R(x)^{2}}\right) \\
& +\psi_{2}(x)=\frac{E^{2} a^{4}}{V_{F}(x)^{2}} \psi_{2}(x),
\end{aligned}
$$

where we use $v \rightarrow x$ for the sake of simplicity. Considering (9), the solutions may take the form

$$
\psi_{1}(x)=f(x) F_{1}(g(x))
$$

Then we have

$$
\begin{aligned}
& F_{1}^{\prime \prime}(x)+\left(\frac{2 f^{\prime}}{f g^{\prime}}+\frac{g^{\prime \prime}}{g^{\prime 2}}+\frac{V_{F}^{\prime}}{V_{F} g^{\prime}}-a \frac{R^{\prime}}{R^{2} g^{\prime}}\right) F_{1}^{\prime}(x) \\
& +\left(\frac{E^{2} a^{4} / V_{F}^{2}-U_{1}(x)}{g^{\prime 2}}+\frac{f^{\prime}}{f g^{\prime 2}}\left(\frac{V_{F}^{\prime}}{V_{F}}-\frac{a R^{\prime}}{R^{2}}\right)\right. \\
& \left.+\frac{f^{\prime \prime}}{f g^{\prime 2}}\right) F_{1}(x)=0 .
\end{aligned}
$$

Equation (12) can also be expressed as

$$
F_{1}^{\prime \prime}(g)+\mathbf{Q}(\mathbf{g}) F_{1}^{\prime}(g)+\mathbf{R}(\mathbf{g}) F_{1}(g)=0,
$$


where

$$
\begin{aligned}
\mathbf{Q}(\mathbf{g}(\mathbf{x}))= & \frac{2 f^{\prime}}{f g^{\prime}}+\frac{g^{\prime \prime}}{g^{\prime 2}}+\frac{V_{F}^{\prime}}{V_{F} g^{\prime}}-a \frac{R^{\prime}}{R^{2} g^{\prime}} \\
\mathbf{R}(\mathbf{g}(\mathbf{x}))= & \frac{E^{2} a^{4} / V_{F}^{2}-U_{1}(x)}{g^{\prime 2}}+\frac{f^{\prime}}{f g^{\prime 2}}\left(\frac{V_{F}^{\prime}}{V_{F}}-\frac{a R^{\prime}}{R^{2}}\right) \\
& +\frac{f^{\prime \prime}}{f g^{\prime 2}} .
\end{aligned}
$$

We may get $U(x)$ as follows:

$$
\begin{aligned}
U_{1}(x)= & \frac{k^{2} a^{4}}{R^{4}}-\frac{2 k R^{\prime}(x) a^{2}}{R(x)^{3}}-\frac{R^{\prime}(x)^{2} a^{2}}{4 R(x)^{4}}-\frac{R^{\prime}(x)^{2} a}{R(x)^{3}} \\
& +\frac{k V_{F}^{\prime}(x) a^{2}}{R(x)^{2} V_{F}(x)}+\frac{R^{\prime}(x) V_{F}^{\prime}(x) a}{2 R(x)^{2} V_{F}(x)} \\
& +\frac{R^{\prime \prime}(x) a}{2 R(x)^{2}} .
\end{aligned}
$$

One can make the coefficient of $F_{1}^{\prime}(g)$ zero and $f(x)$ can be found as

$$
f(x)=\frac{C_{1} e^{-a / 2 R(x)}}{\sqrt{g^{\prime}(x) V_{F}(x)}} .
$$

Then, using $V_{F}(x)=1 / g^{\prime}(x)$ in (16), (13) becomes

$$
F_{1}^{\prime \prime}(x)+\left(a^{4} E^{2}-V_{1}(x)\right) F_{1}(x)=0,
$$

where

$$
V_{1}(x)=\frac{a^{4} k^{2}}{R^{4}(x) g^{\prime 2}}-\frac{2 a^{2} k R^{\prime}(x)}{R^{3}(x) g^{\prime 2}}-\frac{a^{2} k g^{\prime \prime}}{R^{2}(x) g^{\prime 3}} .
$$

In this work [19], it is shown that, with a superpotential $W(x)$ which is a hyperbolic function

$$
W(x)=A \operatorname{coth} x+B \operatorname{csch} x-\frac{\sinh x}{\cosh x+C},
$$

then, the pair of potentials is given as

$$
\begin{aligned}
V_{1}^{(+)}(x)= & \left(A(A+1)+B^{2}\right) \operatorname{csch}^{2} x \\
& +(2 A+1) B \operatorname{csch} x \operatorname{coth} x \\
& +\frac{(2 A-1) C-2 B}{C+\cosh x}+(A-1)^{2}+E \\
V_{1}^{(-)}(x)= & \left(A(A-1)+B^{2}\right) \operatorname{csch}^{2} x \\
& +(2 A-1) B \operatorname{csch} x \operatorname{coth} x \\
& +\frac{(2 A-3) C-2 B}{C+\cosh x}+\frac{2\left(C^{2}-1\right)}{(C+\cosh x)^{2}} \\
& +(A-1)^{2}+E,
\end{aligned}
$$

where this potential is defined on the half line $0<x<$ $\infty$ and is known as generalized Pöschl-Teller potential [2022]. In [22], the authors removed the rational term in $V^{+}(x)$ using a parameter condition $C=2 B /(2 A-1)$. In this case, the potential $V^{+}(x)$ is known as generalized Pöschl-Teller potential in the literature with the exact solutions. Now we will discuss the cases depending on the different choices of rational term in $W(x)$ superpotential. Now we shall look at (18) to arrive at a solvable model given below. If we take

$$
\begin{aligned}
V_{1}(x) & =\frac{a^{4} k^{2}}{R^{4}(x) g^{\prime 2}}-\frac{2 a^{2} k R^{\prime}(x)}{R^{3}(x) g^{\prime 2}}-\frac{a^{2} k g^{\prime \prime}}{R^{2}(x) g^{\prime 3}} \\
& =V_{1} \csc ^{2} x+V_{2} \cot x \csc x+\varepsilon,
\end{aligned}
$$

where $V_{1}, V_{2}, \varepsilon$ are real constants, hence, our function $g(x)$ satisfying (22) can be found as

$$
\begin{aligned}
& g(x)=C_{1} \\
& \quad \pm \int^{x} \frac{i \exp \left(\int^{y}\left(\left(a^{2} k-2 R(z) R^{\prime}(z)\right) / R^{2}(z)\right) d z\right)}{\sqrt{\mu(y)-C_{2}}} d y,
\end{aligned}
$$

where $C_{1}, C_{2}$ are constants and

$$
\mu(y)=2 \int^{y}-\frac{\exp \left(2 \int^{t}\left(\left(a^{2} k-2 R(z) R^{\prime}(z)\right) / R^{2}(z)\right) d z\right)\left(2 V_{1}+\varepsilon+V_{2} \cos t+\varepsilon \cos 2 t \csc ^{2} t R^{2}(t)\right)}{2 a^{2} k} d t .
$$

Now we will search for the solutions for our systems in case of the equal and inequal torus radii.

2.1. Equal Inner and Outer Radii $(a=c)$. First we assume that the superpotential $W_{1}(x)$ is a trigonometric function which is

$$
W_{1}(x)=A \cot x+B \csc x+\frac{\lambda \sin x}{c+a \cos x}
$$

and we get the partner potentials

$$
\begin{aligned}
V_{1}^{-}(x)= & W_{1}^{2}(x)-W_{1}^{\prime}(x) \\
= & (1+2 A) B \cot x \csc x \\
& +\left(A+B^{2}+A^{2}\right) \csc ^{2} x-A^{2} \\
& +\frac{\lambda(-a+\lambda) \sin ^{2} x}{(c+a \cos x)^{2}} \\
& +\frac{\lambda(2 B+(2 A-1) \cos x)}{c+a \cos x}
\end{aligned}
$$




$$
\begin{aligned}
V_{1}^{+}(x)= & W_{2}^{2}(x)+W_{2}^{\prime}(x) \\
= & (2 A-1) B \cot x \csc x \\
& +\left(B^{2}-A+A^{2}\right) \csc ^{2} x-A^{2} \\
& +\frac{\lambda(a+\lambda) \sin ^{2} x}{(c+a \cos x)^{2}}
\end{aligned}
$$$$
+\frac{\lambda(2 B+(2 A+1) \cos x)}{c+a \cos x}
$$

It can be seen from (26) that, in case of an applied special condition $a=1, \lambda=1$, and $x \rightarrow i x$, one can obtain system (20). In order to find a parameter condition on the rational term, we can simplify (26) as

$$
\begin{aligned}
V_{1}^{-}(x)=(1+2 A) B \cot x \csc x+\left(A+B^{2}+A^{2}\right) \csc ^{2} x-A^{2}+\lambda \\
\cdot \frac{2 a(A-1)+4 B c+\lambda+(4 a B-2 c+4 A c) \cos x+(2 a A-\lambda) \cos 2 x}{2(c+a \cos x)^{2}}
\end{aligned}
$$

Then, we can find these conditions as

$$
\begin{aligned}
& \lambda=2 a A \\
& c=\frac{2 a B}{1-2 A} \\
& A=\frac{1}{2}(1 \pm 2 B) \\
& a=|c| .
\end{aligned}
$$

Thus, we have

$$
\begin{aligned}
V_{1}^{-}(x)= & (1+2 A) B \cot x \csc x \\
& +\left(A+B^{2}+A^{2}\right) \csc ^{2} x-A^{2}
\end{aligned}
$$

which is known as trigonometric Pöschl-Teller potential. On the other hand, we can get the partner potential $V_{1}^{+}(x)$ as

$$
\begin{aligned}
V_{1}^{+}(x)= & 2 B^{2} \cot x \csc x+\left(B^{2}+A(A-1)\right) \csc ^{2} x \\
& +(2 B+1) \csc ^{2} \frac{x}{2}-A^{2} .
\end{aligned}
$$

Here, it is well known that (33) and (34) are isospectral partner potentials except the ground state. Comparing (33) and $V_{1}(x)$ in (22), we obtain

$$
\begin{aligned}
V_{1}(x) & =V_{1}^{-}(x), \\
V_{1} & =(1+2 A) B, \\
V_{2} & =A(A+1)+B^{2}, \\
\varepsilon & =-A^{2} .
\end{aligned}
$$

The solutions of $V_{-}(x)$ are already known as [20]

$$
E_{1, n}^{-}= \pm \frac{\sqrt{(n-\lambda / 2 a)^{2}-\lambda^{2} / 4 a^{2}}}{a^{2}} .
$$

This result shows that when $a \rightarrow \infty, E \rightarrow 0$. Keeping in mind the eigenvalue equation (17), we can get

$$
\begin{aligned}
\widehat{H}_{-} F_{1, n}^{(-)}(x) & =E^{2} a^{4} F_{1, n}^{(-)}(x), \\
\widehat{H}_{-} & =\widehat{A}^{\dagger} \widehat{A}, \\
\widehat{A} & =\frac{d}{d x}+W_{1}(x) .
\end{aligned}
$$

Then, the solutions are written as [20]

$$
\begin{aligned}
F_{1, n}^{(-)}(x) \sim & (1-\cos x)^{(-A-B) / 2}(1+\cos x)^{(-A+B) / 2} \\
& \cdot P_{n}^{(-A-B-1 / 2,-A+B-1 / 2)} .
\end{aligned}
$$

In fact, the complete solutions are not given yet. The spinor solutions can be given as

$$
\begin{aligned}
\psi_{1}, n(x)= & N_{1} \exp \left(-\frac{a}{2(a+a \cos x)}\right) \\
& \cdot(1-\cos x)^{(-A-B) / 2}(1+\cos x)^{(-A+B) / 2} \\
& \cdot P_{n}^{(-A-B-1 / 2,-A+B-1 / 2)}(\cos x)
\end{aligned}
$$

where $N_{1}$ is the normalization constant. The solutions $F_{+}(x)$ corresponding to the ones for the partner potential $V_{1,+}(x)$ can be found. The system can be summarized as follows:

$$
\begin{aligned}
\widehat{H}_{+} F_{1}^{+}(x) & =E^{2} a^{4} F_{1}^{+}(x), \\
H_{+} & =\widehat{A} \widehat{A}^{\dagger} .
\end{aligned}
$$

For (34),

$$
F_{1, n}^{(+)}=\frac{1}{\sqrt{E_{n,-}}} \widehat{A} F_{1, n}^{(-)}
$$

and it is known that the energy states of these Hamiltonians are given by

$$
E_{1, n+1}^{-}=E_{2, n}^{+} \text {. }
$$


At the same time, we can find $F_{1, n}^{(+)}(x)$ solutions using (41):

$$
\begin{aligned}
& F_{1, n}^{(+)}(x) \\
& \quad \sim \frac{(1-\cos x)^{(-A-B) / 2}(1+\cos x)^{(1 / 2)(B-A)}}{a}\left(\frac{a(2 A-n)}{2}\right. \\
& \cdot P_{n-1}^{(1 / 2-A-B, 1 / 2-A+B)}(\cos x) \sin x \\
& \left.+\lambda P_{n}^{(-1 / 2-A-B,-1 / 2-A+B)}(\cos x) \tan \frac{x}{2}\right) .
\end{aligned}
$$

When it comes to the solutions $\psi_{2}(x)$ of (10) which shares the same energy with (9), we get

$$
\begin{aligned}
U_{2}(x)= & \frac{k^{2} a^{4}}{R^{4}}+\frac{2 k R^{\prime}(x) a^{2}}{R(x)^{3}}-\frac{R^{\prime}(x)^{2} a^{2}}{4 R(x)^{4}} \\
& -\frac{R^{\prime}(x)^{2} a}{R(x)^{3}}-\frac{k V_{F}^{\prime}(x) a^{2}}{R(x)^{2} V_{F}(x)} \\
& +\frac{R^{\prime}(x) V_{F}^{\prime}(x) a}{2 R(x)^{2} V_{F}(x)}+\frac{R^{\prime \prime}(x) a}{2 R(x)^{2}} .
\end{aligned}
$$

$$
\begin{aligned}
& g_{2}(x)=C_{2} \\
& \quad \pm \int^{x} \frac{i \exp \left(-\int^{u}\left(\left(a^{2} k+2 R(y) R^{\prime}(y)\right) / R^{2}(y)\right) d y\right)}{\sqrt{-C_{1}+2 \int^{u}\left(\left(\exp \left(-2 \int^{y}\left(\left(a^{2} k+2 R(y) R^{\prime}(y)\right) / R^{2}(y)\right) d y\right)\left(2 P_{1}+\varepsilon+2 P_{2} \cos z-\varepsilon \cos 2 z\right) R^{2}(z) \csc ^{2} z\right) / 2 a^{2} k\right) d z}} d u .
\end{aligned}
$$

If the parameters are chosen as $P_{1}=V_{2}$ and $P_{2}=-V_{1}$, then we get

$$
\begin{aligned}
V_{2}(x)= & -A^{2}-B(1+2 A) \cot x \csc x \\
& +\left(A(A+1)+B^{2}\right) \csc ^{2} x,
\end{aligned}
$$

and using (29), $P_{1}$ and $P_{2}$ can be expressed in terms of the torus parameter and hence, one can get the supersymmetric partner potentials for $V_{2}(x)$ which are $V_{2}^{ \pm}$:

$$
\begin{aligned}
V_{2}^{-}(x)= & V_{2}(x) \\
= & -\frac{\lambda^{2}}{4 a^{2}}-\left(\frac{1}{2}-\frac{\lambda^{2}}{2 a^{2}}\right) \cot x \csc x \\
& +\frac{1}{2}\left(\frac{1}{2}+\frac{\lambda^{2}}{a^{2}}\right) \csc ^{2} x, \\
V_{2}^{+}(x)= & 2 B^{2} \cot x \csc x+\left(B^{2}+A(A-1)\right) \csc ^{2} x \\
& +(1-2 B) \csc ^{2} \frac{x}{2}-A^{2}
\end{aligned}
$$

The wavefunction mapping can be given as

$$
\psi_{2}(x)=f(x) F_{2}\left(g_{2}(x)\right)
$$

and we obtain

$$
\begin{aligned}
F_{2}^{\prime \prime}(x)+\left(a^{4} E^{2}-V_{2}(x)\right) F_{2}(x) & =0 \\
V_{F}(x) & =\frac{1}{g_{2}^{\prime}(x)},
\end{aligned}
$$

where

$$
V_{2}(x)=\frac{a^{4} k^{2}}{R^{4}(x) g_{2}^{\prime 2}}+\frac{2 a^{2} k R^{\prime}(x)}{R^{3}(x) g_{2}^{\prime 2}}+\frac{a^{2} k g_{2}^{\prime \prime}}{R^{2}(x) g_{2}^{\prime 3}}
$$

Then, we can get $V_{2}(x)$ as

$$
V_{2}(x)=\varepsilon+P_{2} \cot x \csc x+P_{1} \csc ^{2} x,
$$

where $P_{1}$ and $P_{2}$ are real constants and equating (47) and (48), $g_{2}(x)$ is found to be 


$$
\begin{aligned}
V_{1}^{-}(x)= & B(1+2 A) \cot x \csc x+\left(A^{2}+A+B^{2}\right) \csc ^{2} x-A^{2} \\
& +\frac{G(x)^{2}+(2(c+a \cos x)(B+A \cos x) \csc x-a \sin x) G(x)+(c+a \cos x) G^{\prime}(x)}{(c+a \cos x)^{2}} .
\end{aligned}
$$

In order to make the rational term zero, $G(x)$ can be found as follows:

$$
G(x)=\frac{c+a \cos x}{(\sin x)^{-2 A+2 B}(1-\cos x)^{-2 B}\left(4^{-A}+4^{B} \mathscr{B}\left(\cos ^{2}(x / 2)\right) ; 1 / 2+A-B, 1 / 2+A+B\right)},
$$

where $\mathscr{B}(z ; s, w)$ is the incomplete beta function which is equal to

$$
\mathscr{B}(z ; s, w)=\int^{z} u^{s-1}(1-u)^{w-1} d u, \quad z \in(0,1)
$$

$$
\begin{aligned}
V_{1}^{-}(x)= & B(1+2 A) \cot x \csc x+\left(A^{2}+A+B^{2}\right) \csc ^{2} x-A^{2}, \\
V_{1}^{+}(x)= & (2 A-1) B \cot x \csc x+\left(A^{2}-A+B^{2}\right) \csc ^{2} x-A^{2} \\
& +\frac{\left(\mathscr{B}\left(\cos ^{2} x / 2,1 / 2+A-B, 1 / 2+A+B\right) \xi(x)+\chi(x)\right)(\sin (x / 2))^{-1+2 A+2 B}}{2^{2 A+2 B} \mathscr{B}\left(\cos ^{2} x / 2,1 / 2+A-B, 1 / 2+A+B\right)(\sin x)^{2 A+2 B}+2^{-2 A-2 B} C_{1}(\sin (x / 2))^{2 A+2 B}(\sin (x / 2))^{2 A+2 B}},
\end{aligned}
$$

where

$$
\begin{gathered}
\xi(x)=2^{3 / 2+8 A}\left(\cos \frac{x}{2}\right)^{6 A+2 B} \sqrt{1-\cos x}(B+A \cos x) \\
\cdot \csc x\left(\sin \frac{x}{2}\right)^{4 A+4 B} \\
\chi(x)=2^{-1 / 2+4 A}\left(\cos \frac{x}{2}\right)^{6 A}\left(\sin \frac{x}{2}\right)^{4 A+4 B} \\
\cdot\left(4 C_{1}\left(\cos \frac{x}{2}\right)^{2 B} \sqrt{1-\cos x}(B+A \cos x) \csc x\right.
\end{gathered}
$$

$$
\begin{aligned}
& +16^{A}\left(\cos \frac{x}{2}\right)^{2 A}\left(\sin \frac{x}{2}\right)^{2 A+2 B} \\
& \left.\cdot\left(\sqrt{1-\cos x}+\sqrt{1+\cos x} \tan \frac{x}{2}\right)\right) .
\end{aligned}
$$

For the unknown $G(x)$ case, there are no restrictions for the parameters. Equations (57) and (58) share the same spectrum except the ground state which is

$$
E_{1, n}^{-}= \pm \frac{\sqrt{(n-A)^{2}-A^{2}}}{a^{2}}
$$

On the other hand, we can get an ansatz for $W(x)$ :

$$
\begin{aligned}
W(x)= & A \cot x+B \csc x+\frac{G(x)+\lambda \sin x}{c+a \cos x}, \\
V_{1}^{-}(x)= & B(2 A+1) \cot x \csc x+\left(A^{2}+A+B^{2}\right) \csc ^{2} x-A^{2} \\
& +\frac{\lambda(2 a(A-1)+4 B c+\lambda)+2 \lambda \cos x(2 a B+c(2 A-1))+(2 a A-\lambda) \cos 2 x+\mathscr{G}(x)}{2(c+a \cos x)^{2}},
\end{aligned}
$$


where

$$
\begin{aligned}
\mathscr{G}(x)= & 2 G(x)^{2}+G(x)(4 a B+4 A c) \cot x \\
& +4 a A \cos x \cot x+4 B c \csc x \\
& +(4 \lambda-2 a) \sin x-2(c+a \cos x) G^{\prime}(x) .
\end{aligned}
$$

If we terminate the rational term in (62), we find

$$
\begin{aligned}
1+2 a(A-1)+4 B c+\lambda & =0, \\
4 a B-2 c+4 A c & =0, \\
2 a A-\lambda & =0, \\
\mathscr{G}(x) & =0,
\end{aligned}
$$

and we can also obtain

$$
\begin{aligned}
& A=\frac{\lambda}{2 A}, \\
& B=\frac{1}{2}\left( \pm \frac{\sqrt{1-2 a+2 \lambda}}{\sqrt{-2 a+2 \lambda}}-\frac{\lambda \sqrt{1-2 a+2 \lambda}}{a \sqrt{-2 a+2 \lambda}}\right), \\
& c= \pm \frac{a \sqrt{1-2 a+2 \lambda}}{\sqrt{-2 a+2 \lambda}} .
\end{aligned}
$$

To terminate the remaining function on the nominator of (62), one can take $\mathscr{G}(x)=0$; hence $G(x)$ is obtained as

$$
\begin{aligned}
& G(x) \\
& =\frac{8^{A}(1+\cos x)^{A}(c+a \cos x)}{C_{1}(\cot (x / 2))^{2 B}(c+a \cos x)^{2 \lambda / a}(\sin (x / 2))^{-2 A} \varrho(x)},
\end{aligned}
$$

where

$$
\begin{aligned}
& \varrho(x)=-\frac{1}{1+2 A+2 B} 2^{4 A+1 / 2} \operatorname{Appel} F_{1}\left(\frac{1}{2}+A\right. \\
& +B, \frac{1}{2}-A+B, \frac{2 \lambda}{a}, \frac{3}{2}+A \\
& \left.+B, \frac{\sin ^{2} x}{2}, \frac{a-a \cos x}{a+c}\right)\left(\cos \frac{x}{2}\right)^{2 B} \\
& \cdot \sqrt{1+\cos x}\left(\frac{c+a \cos x}{a+c}\right)^{2 \lambda / a} \tan \frac{x}{2} .
\end{aligned}
$$

Here, $F_{1}\left(\alpha, \beta, \beta^{\prime}, \gamma ; x ; y\right)$ is the Appel hypergeometric function with two variables. Thus, $V_{1,-}(x)$ can be given by (57) and $V_{+}(x)$ becomes

$$
\begin{aligned}
V_{1}^{+}(x)= & (2 A-1) B \cot x \csc x+\left(B^{2}+A^{2}-A\right) \csc ^{2} x-A^{2}+\lambda \\
& \cdot \frac{2 a(A+1)+4 B c+\lambda+2(2 a B+c+2 A c) \cos x+(2 a A-\lambda) \cos 2 x}{2(c+a \cos x)^{2}} \\
& +\frac{\chi(x)}{(c+a \cos x)\left(C_{1}(\cot (x / 2))^{2 B}(c+a \cos x)^{2 \lambda / a}\right)-\varrho(x)},
\end{aligned}
$$

where

$$
\begin{aligned}
& \chi(x)=\left(8 ^ { \lambda } \left((1+\cos x)^{A}\right.\right. \\
& . \frac{2 \varrho(x)(a A+2 B c+\lambda+2(a B+A c) \cos x+(a A-\lambda) \cos 2 x)}{\sqrt{1+\cos x}} \\
& +2\left(8^{A}(1+\cos x)^{A}\right)(c+a \cos x)+C_{1}\left(\cos \frac{x}{2}\right)^{2 B} \\
& \cdot(c+a \cos x)^{2 \lambda / a} \\
& \cdot(a A+2 B c+\lambda+2(a B+A c) \cos x+(a A-\lambda) \cos 2 x) \csc x \\
& \left.\left.\cdot\left(\sin \frac{x}{2}\right)^{-2 A-2 B}\right)\right) .
\end{aligned}
$$

Similarly, one can obtain the partner potentials for the system given in (10).

\section{The iso(2, 1) Algebraic Approach}

Let us look at the operators given below:

$$
\begin{aligned}
J_{ \pm} & =i e^{ \pm i \phi}\left( \pm \frac{\partial}{\partial x}-\left(\left(-i \frac{\partial}{\partial x} \pm \frac{1}{2}\right) S(x)-T(x)\right)\right. \\
& \left.+U\left(x, \mu \pm \frac{1}{2}\right)\right) \\
J_{3} & =-i \frac{\partial}{\partial \phi} .
\end{aligned}
$$

Here, the term $U(x, \mu \pm 1 / 2)$ is the modification operator which is used in [22] and $\mu$ is a constant. Without $U(x, \mu \pm$ $1 / 2)$, these operators are known as those given in the iso $(2,1)$ algebra but this functional operator, $U(x, \mu \pm 1 / 2)$, helps to construct the algebra for the rationally extended potentials. Here, we discuss the extended trigonometric Pöschl-Teller 
potential within the iso $(2,1)$ algebra. These operators provide the commutation relations which are given as

$$
\begin{aligned}
& {\left[J_{+}, J_{-}\right]=-2 J_{3},} \\
& {\left[J_{3}, J_{ \pm}\right]= \pm J_{ \pm} .}
\end{aligned}
$$

If $J_{ \pm}$and $J_{3}$ are used in (71), then, one can get

$$
\begin{aligned}
S^{\prime}(x)-S(x)^{2} & =1, \\
T^{\prime}(x)-S(x) T(x) & =0 .
\end{aligned}
$$

The constraints in (71) also lead to [22]

$$
\begin{aligned}
& U_{1}(x)^{2}-\frac{d}{d x} U_{1}(x)+2 U_{1}(x)\left(F(x)\left(\mu+\frac{1}{2}\right)\right. \\
& -G(x))-\left(U_{2}(x)^{2}-\frac{d}{d x} U_{2}(x)\right. \\
& \left.+2 U_{2}(x)\left(F(x) \mu_{1}-G(x)\right)\right)=0 .
\end{aligned}
$$

In this study, we will use $U(x, \mu+1 / 2)=U_{1}(x)$ and $U(x, \mu-$ $1 / 2)=U_{2}(x)$. And the Hamiltonian in (37) can be given in terms of the Casimir operator $J^{2}=J_{3}^{2}-(1 / 2)\left(J_{+} J_{-}+J_{-} J_{+}\right)$, and we may denote the Hamiltonian using $J^{2}$ as

$$
H=J^{2}+\frac{1}{4}
$$

Here, the operators act on the physical states which are given by

$$
\begin{aligned}
& J^{2}|j, \mu\rangle=j(j+1)|j, \mu\rangle \\
& J_{3}|j, \mu\rangle=\mu|j, \mu\rangle \\
& J_{ \pm}|j, \mu\rangle=\sqrt{-(j \mp \mu)(j \pm \mu+1)}|j, \mu \pm 1\rangle .
\end{aligned}
$$

And, $|j, \mu\rangle$ can be written in the function space as

$$
|j, \mu\rangle=\psi_{j \mu}(x) e^{i \mu \phi} .
$$

If we express $J^{2}$ using the operators as [22]

$$
\begin{aligned}
J^{2}= & -\frac{d^{2}}{d x^{2}}+\left(1+F(x)^{2}\right)\left(J_{3}^{2}-\frac{1}{4}\right)-2 G^{\prime}(x)\left(J_{3}\right) \\
& +G(x)^{2}-\frac{1}{4}+U_{1}^{2}(x) \\
& +\left(\left(J_{3}+\frac{1}{2}\right) F(x)-G(x)\right) U_{1}(x) \\
& +U_{1}(x)\left(\left(J_{3}+\frac{1}{2}\right) F(x)-G(x)\right)-\frac{d}{d x} U_{1}(x) \\
= & -\frac{d^{2}}{d x^{2}}+V(x),
\end{aligned}
$$

choosing our functions $S(x)$ and $T(x)$ as

$$
\begin{aligned}
& S(x)=-\cot x, \\
& T(x)=B_{1} \csc x,
\end{aligned}
$$

using a suggestion for each $U_{1}(x), U_{2}(x)$ which are given by

$$
\begin{aligned}
& U_{1}(x)=-\frac{K_{1} \sin x}{c+a \cos x}, \\
& U_{2}(x)=\frac{K_{2} \sin x}{c+a \cos x},
\end{aligned}
$$

and using $U_{1}(x)$ in (77), we can find the potential which is an element of $J^{2}$ :

$$
\begin{aligned}
V(x)= & -\frac{1}{4}+2 B_{1} \mu \cot x \csc x \\
& +\left(\mu^{2}+B_{1}^{2}-\frac{1}{4}\right) \csc ^{2} x \\
& +\frac{2 K_{1}\left(B_{1}+(\mu+1) \cos x\right)}{c+a \cos x} \\
& +\frac{K_{1}\left(a+K_{1}\right) \sin ^{2} x}{(c+a \cos x)^{2}} .
\end{aligned}
$$

For the $a=c$ case, we can compare (80) and (26); then we get,

$$
\begin{aligned}
& \lambda=-K_{1}, \\
& B=-B_{1}, \\
& A=-\mu-\frac{1}{2} .
\end{aligned}
$$

Moreover, (73) can be satisfied in case of chosen $U_{1}(x)$ and $U_{2}(x)$ in (79) and the parameter conditions as given below:

$$
\begin{aligned}
B_{1} & =-\frac{c+K_{1}}{2 c}, \\
\mu & =\frac{K_{1}}{2 c}-\frac{1}{2}, \\
a & =c, \\
K_{2} & =-K_{1}-2 c, \\
\mu_{1} & =\mu+1 .
\end{aligned}
$$

Thus, the energy eigenvalues can be expressed in terms of the parameters given above:

$$
E_{n}= \pm \frac{1}{a} \sqrt{\left(n+\mu+\frac{1}{2}\right)^{2}-\left(\mu+\frac{1}{2}\right)^{2}}
$$

where one can say that $j=n+\mu$ to compare our results with those found in [22]. 


\section{Conclusions}

Our findings in this paper point to the fact that the exact solutions for a given system which is relativistic can be obtained using the similar techniques used in nonrelativistic quantum mechanics. Especially considering the massless particle dynamics, the latest trends in relativistic quantum mechanics can bring about new bound state problems which are not solved yet such as the Dirac equation in curved spacetime which has a toroidal geometry. Because the metric contains a more general trigonometric function which is $R(x)=c+a \cos x$, the Klein-Gordon-like equations obtained from the couple of first-order Dirac equations are not the familiar ones which are generally known in relativistic quantum mechanics. In this problem, the Fermi velocity is chosen as a nonconstant function which is expressed in terms of the point transformation function in our solutions; after that, solvable potentials are derived using the superpotential suggestions. With equal inner and outer radius case, one of the partner potentials is trigonometric Pöschl-Teller potential while the other one is including the extra trigonometric function $\csc (x / 2)$. We have obtained the solutions of the partner potentials for each of systems (9) and (10); for the different radius values of the torus surface, as a more general case, one of the partner potentials is found as nonsolvable rational function which includes beta function while the other one is known Pöschl-Teller potential. In the next case, unsolvable partner potential whose partner is again PöschlTeller potential is given in terms of the Appel hypergeometric function. In the final section of this work, operators of the Lie algebra iso $(2,1)$ are found in order to express the Casimir operator with the potential functions and the extended trigonometric Pöschl-Teller potentials which are (26) and (27) given in the $a=c$ case. Finally, we note that the Dirac equation on the toroidal spacetime problem can lead to obtaining more general potential families.

\section{Conflicts of Interest}

The author declares that there are no conflicts of interest regarding the publication of this paper.

\section{References}

[1] P. A. M. Dirac, "The quantum theory of the electron," Proceedings of the Royal Society A Mathematical, Physical and Engineering Sciences, vol. 117, no. 778, pp. 610-624, 1928.

[2] L. Parker, "One-electron atom in curved space-time," Physical Review Letters, vol. 44, no. 23, pp. 1559-1562, 1980.

[3] G. A. de Marques and V. B. Bezerra, "Some effects on relativistic quantum systems due to a weak gravitational field," Brazilian Journal of Physics, vol. 35, no. 4, p. 1678, 2005.

[4] F. Finster, S. Murro, and C. Röken, "The fermionic signature operator and quantum states in Rindler space-time," Journal of Mathematical Analysis and Applications, vol. 454, no. 1, pp. 385411, 2017.

[5] N. Kamran and R. G. McLenaghan, "Symmetry operators for neutrino and Dirac fields on curved spacetime," Physical Review D: Particles, Fields, Gravitation and Cosmology, vol. 30, no. 2, pp. 357-362, 1984.
[6] M. V. Gorbatenko and V. P. Neznamov, "Solution of the problem of uniqueness and Hermiticity of Hamiltonians for Dirac particles in gravitational fields," Physical Review D: Particles, Fields, Gravitation and Cosmology, vol. 82, no. 10, Article ID 104056, 2011.

[7] F. Safari, H. Jafari, J. Sadeghi, S. J. Johnston, and D. Baleanu, "Stability of Dirac equation in four-dimensional gravity," Chinese Physics Letters, vol. 34, no. 6, Article ID 060301, 2017.

[8] Ö. Yeşiltaş, "Non-hermitian dirac hamiltonian in threedimensional gravity and pseudosupersymmetry," Advances in High Energy Physics, vol. 2015, Article ID 484151, 8 pages, 2015.

[9] A. V. Zhukov, R. Bouffanais, N. N. Konobeeva, and M. B. Belonenko, "On the electronic spectrum in curved graphene nanoribbons," Solid State Communications, vol. 151, no. 1147, 2011.

[10] F. Cardin and L. Zanelli, "The geometry of the semiclassical wave front set for Schrödinger eigenfunctions on the torus," Mathematical Physics, Analysis and Geometry, vol. 20, no. 10, 2017.

[11] L. Zanelli, "Schrodinger spectra and the effective Hamiltonian of weak KAM theory on the flat torus," Journal of Mathematical Physics, vol. 57, no. 8, Article ID 081507, 2016.

[12] L. Schneiderbauer and H. C. Steinacker, "Measuring finite quantum geometries via quasi-coherent states," Journal of Physics A: Mathematical and General, vol. 49, no. 28, 285301, 44 pages, 2016.

[13] A. Schulze-Halberg and B. Roy, "Generalized quantum nonlinear oscillators: Exact solutions and rational extensions," Journal of Mathematical Physics, vol. 57, no. 10, Article ID 102103, 2016.

[14] R. K. Yadav, N. Kumari, A. Khare, and B. P. Mandal, "Group theoretic approach to rationally extended shape invariant potentials," Annals of Physics, vol. 359, pp. 46-54, 2015.

[15] A. Schulze-Halberg and B. Roy, "Darboux partners of pseudoscalar Dirac potentials associated with exceptional orthogonal polynomials," Annals of Physics, vol. 349, pp. 159-170, 2014.

[16] O. Mustafa, "Exact and approximate solutions of Schrödinger's equation for a class of trigonometric potentials," Central European Journal of Physics, vol. 11, no. 4, pp. 37-48, 2013.

[17] H. Weyl, "Elektron und Gravitation. I," Zeitschrift für Physik, vol. 56, no. 5-6, pp. 330-352, 1929 (German).

[18] V. Fock, "Geometrisierung der Diracschen Theorie des Elektrons," Zeitschrift für Physik, vol. 57, no. 3-4, pp. 261-277, 1929.

[19] B. Bagchi, C. Quesne, and R. Roychoudhury, "Isospectrality of conventional and new extended potentials, second-order supersymmetry and role of PT symmetry," Pramana-Journal of Physics, vol. 73, no. 2, pp. 337-347, 2009.

[20] G. Levái, "A search for the shape-invariant solvable potentials," Journal of Physics A: Mathematical and General, vol. 22, no. 6, pp. 689-702, 1989.

[21] F. Cooper, A. Khare, and U. Sukhatme, "Supersymmetry and quantum mechanics," Physics Reports, vol. 251, no. 5-6, pp. 267385, 1995.

[22] R. K. Yadav, A. Khare, B. Bagchi, N. Kumari, and B. P. Mandal, "Parametric symmetries in exactly solvable real and PT symmetric complex potentials," Journal of Mathematical Physics, vol. 57, no. 6, Article ID 062106, 2016. 

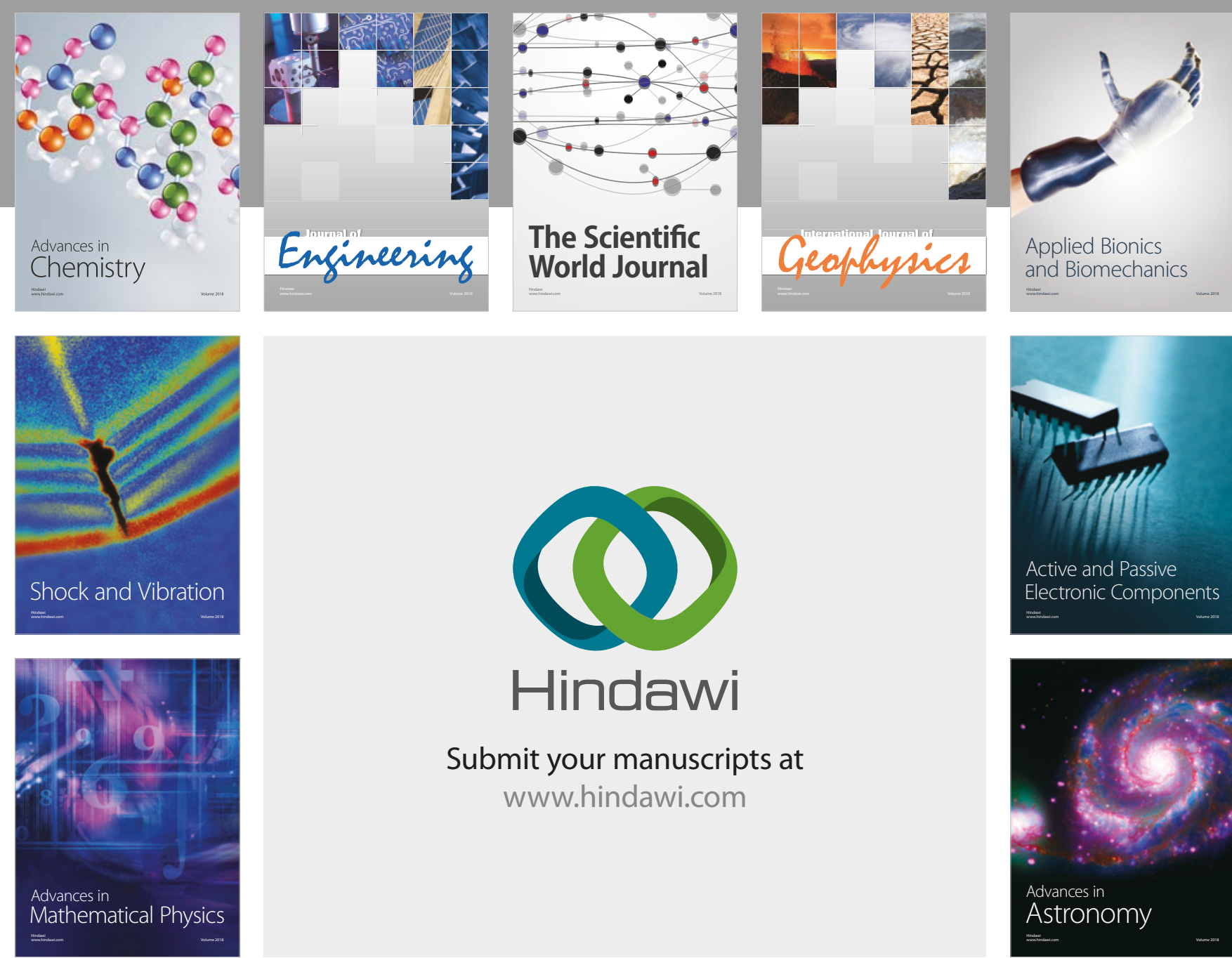

Submit your manuscripts at

www.hindawi.com

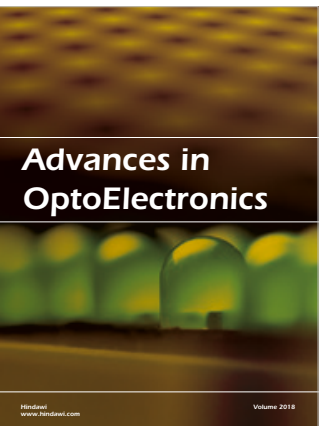

\section{Rotcting Machinery}
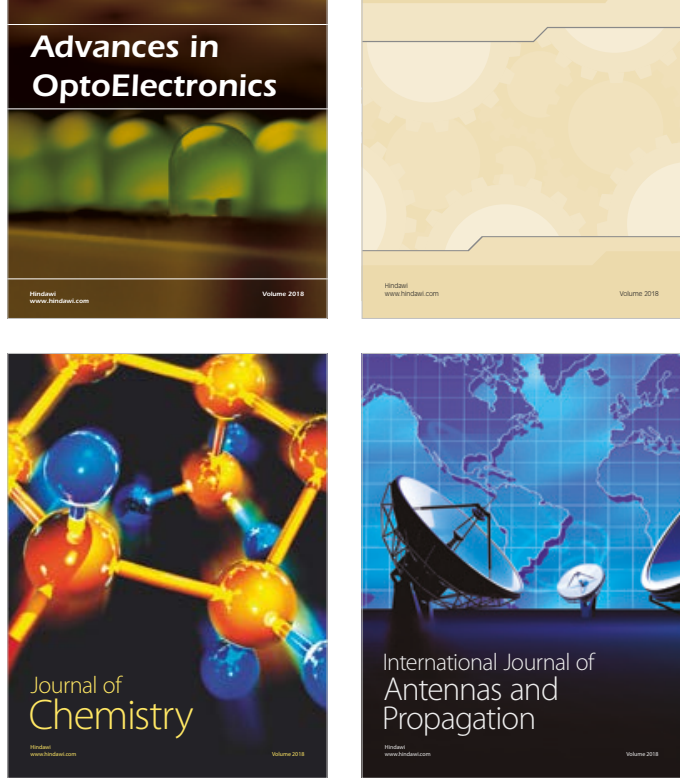

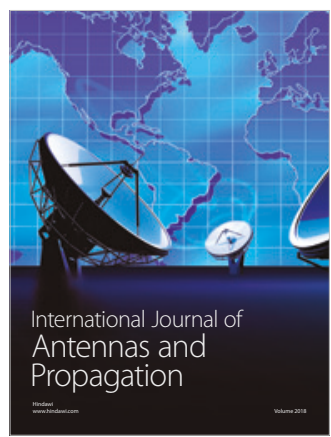

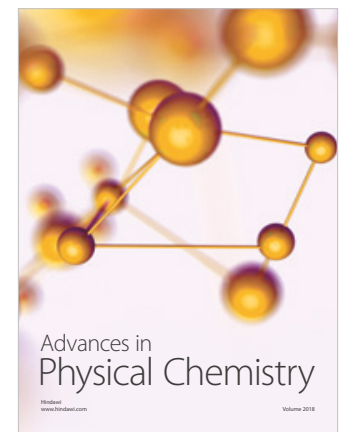

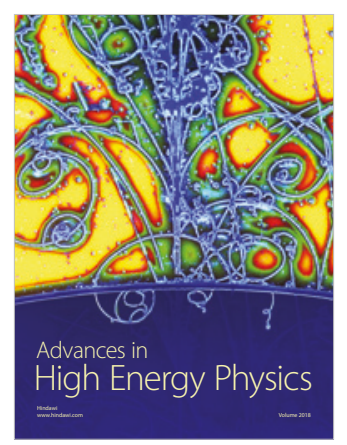

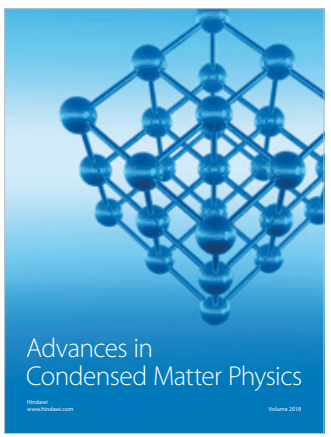

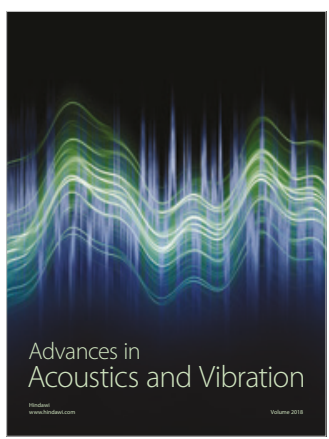

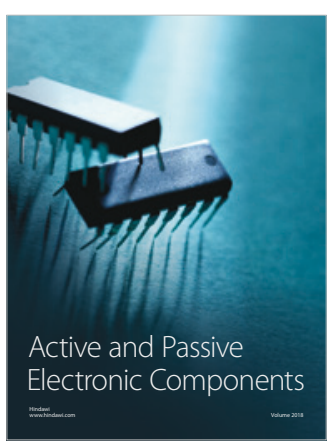
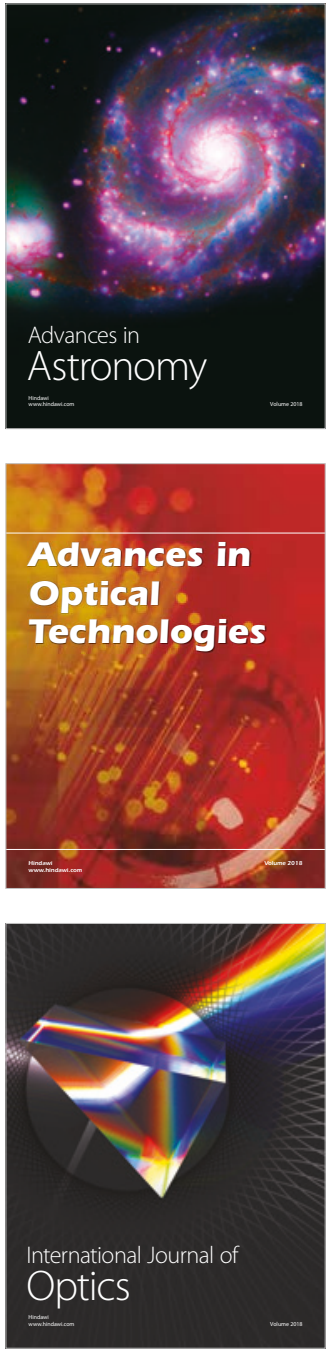